E. Samuelsson (1937). Arsskr. $f$, Alnarps lantbr.,-mejeri-o, tradg. Institut.

F. Sснмiтt et M. E. Schulz (1951). Milchwssenschaft, 6, 189.

M. E. Schulz (1947). Milchwissenschajt, 2, 293.

- (1948). Milchwissenschaft, 3, 196.

- (1949). XIIth Int. Dairy Congr. 1949, 3. 192.

- et W. Schulz (1948). Milchwissenchaft, 3, 213, 253.

A. SMekal (1936). Ergebn. exakt. Naturw., 15, 106.

- (1949). Glastech. Ber., 22, 278.

L. Staible (1948). Süddtsch. Molkereiztg, 69, 59.

A. L. Stigen (1945). Svenska Mejeritidn., 37, 202, 211.

- A. Berglöf et T. Hansson (1949). XIIth Int. Dairy Congr. 1949, 3 139.

A. Stöffelen (1950). Süddtsch. Molkereiztg, 71, 616.

T. Storgards (1951). Karjantuote, 34, 171.

D. Sтüssi (1946). Schweiz. Käserei u. Molkerei Kalender.

- (1948). Süddsch. Molkereiztg, 79, 158.

B. WauschKuHN (1949). Milchwissenschaft, 4, 78.

C. F. Weinzeich (1949). XIIth Int. Dainy Congr. 1949, 12.

O. Wellhausen (1948). Milchwissenschaft, 3, 344.

S. G. Wiechers et B. DE GOede (1950). "Continuous Buttermaking ". Amsterdam : North Holland Publishing Company.

F. Wigan (1949). XIIth Int. Dairy Congr. 1949, 3, 165.

W. J. Wiley et G. Loftus Hills (1950, 1951). Aust. Dairy Rev., 18 (12), 15 ; 19 (1), $15 ; 19(2), 15$.

W. Wilsmann et E. Feltens (1947). Milchwissenschaft, 2, 303.

- et - (1950). Molkerei-u. Käserei-Ztg, Hildesh., 1.

G. Wode (1933). Medd. Centanst. Försöksv. Jordbr., 438 ; Mejeriavd., 46.

\title{
MÉTHODE D'ANALYSE DES LAITS BARATTÉS ET COAGULÉS (1)
}

par

\section{G. CHENEVAL}

Chimiste prineipal à la Station Agronomique de Nantes

Il arrive fréquemment que les laits parviennent au Laboratoire dans un tel état qu'il est bien difficile, pour ne pas dire impossible, de les rendre parfaitement homogènes. Les échantillons peuvent être caillés et plus ou moins altérés suivant l'ancienneté du prélèvement et les précautions prises pour leur conservation. Ils peuvent être seulement barattés; ce fait s'observe surtout pour les laits pasteurisés, et dans ce cas, la matière grasse est à l'état de beurre qu'il est impossible d'incorporer au reste de l'échantillon. Si l'équilibre physique de ces laits barattés est détruit, leur composition chi-

(1) Annales Fals. Fraudes, 1952, 517-518, 24. 
mique n'est pas altérée, leur lactose est intact. Il en est de même pour les laits récemment coagulés avec la réserve de l'augmentation d'acidité, due à la transformation du lactose en acide lactique.

Le problème, pour le chimiste, est done, avant tout, celui d'une bonne homogénéisation. Pour le résoudre. M. VoIret a proposé récemment de combiner l'action de la chaleur et celle de moyens mécaniques (voir A. F., 1950 - p. 5 et p. 30 ).

Tout dernièrement fonctionnait au Laboratoire Municipal de Paris, un homogénéiseur donnant, paraît-il, d'excellents résultats.

Il ne nous a pas été donné d'essayer ces appareils mécaniques qui ne sont pas toujours à la portée de tous les laboratoires appelés à examiner les laits. Et avec les moyens dont nous disposions, nous avons cherché un mode opératoire pratique donnant des résultats suffisamment précis pour pouvoir établir des conclusions.

Après quelques essais infructueux, nous avons vite adopté le point de vue que, pour les laits barattés ou caillés, il était indispensable d'opérer sur la totalité de l'échantillon. L'idée n'est pas nouvelle, et le mérite en revient à KLING, qui publia sa méthode en 1909 (voir Kling et Roy - A. F., 1909, p. 257). Sa technique, il faut bien l'admettre, n'est pas de nature à engendrer un enthousiasme excessif. Elle demande de longues manipulations et ne permet, telle qu'elle est décrite, que le dosage des matières grasse et azotée. Pour se faire une opinion tant soit peu motivée sur la qualité d'un lait, il nous semble indispensable d'en connaître d'autres éléments. On peut y parvenir facilement avec le mode opératoire décrit ci-dessous, dont le principe est fort simple :

Il consiste à effectuer le traitement préconisé pour le dosage du beurre par la méthode d'Adam, non pas sur une prise d'essai de $10 \mathrm{~cm}^{3}$ dans un galactomètre, mais sur la totalité de l'échantillon dans un entonnoir à séparation de un litre.

Quel que soit l'état initial de la matière grasse, on obtient une solution éthérée que l'on amène à un volume connu et qui contient la totalité du beurre. La partie aqueuse et ammoniacale renferme sous forme dissoute ou colloïdale, tous les autres constituants du lait. Elle est amenée également à un volume connu et tous les éléments peuvent y être dosés sur des parties aliquotes.

Cette méthode que nous ne prétendons pas proposer comme une panacée permettant de résoudre parfaitement tous les cas, peut soulever des objections et n'est pas sans mériter quelques critiques.

Il est bien évident qu'elle ne "ressuscitera " pas un lait " pourri " et qu'elle ne permettra pas un dosage de lactose plus précis que toute autre méthode. Mais les nombreux essais effectués nous autorisent à affirmer que tous les autres éléments, même les matières azotées, peuvent être dosés avec une précision guère diffé- 
rente de celle qui est obtenue dans l'examen des laits frais. Et dans les laits peu altérés.: barattés ou fraîchement coagulés, elle permettra une détermination du lactose assez exacte pour pouvoir calculer la constante moléculaire simplifiée dont l'intérêt est indiscutable.

\section{$*^{*} *$}

Voici le mode opératoire que nous avons mis au point :

a) Pesée de l'échantillon. - Le poids total de la bouteille, bouchon compris est déterminé. La bouteille, une fois vidée, nettoyée et égouttée, sera tarée. On aura ainsi le poids total du lait examiné soit : $\mathrm{P}$ gr.

b) Homogénéisation. - Le lait sera ensuite divisé en le faisant passer à travers un tamis ayant des ouvertures d'environ $1 \mathrm{~mm}$. en s'aidant d'une baguette de verre à bout aplati.

La totalité du liquide sera rassemblée dans un vase à précipitation de $350 \mathrm{~cm}^{3}$. Il sera bon de rincer sommairement le matériel employé avec un petit volume d'eau (10 $\mathrm{cm}^{3}$ environ) qui sera réuni au liquide dans le vase à précipițer.

Remarques. - Toutes les fois que l'état d'homogénéisation du mélange global le permettra, au lieu de prendre la totalité du liquide, on pourra sans diluer peser une partie du mélange : 50 ou 100 gr., par exemple, et lui appliquer le traitement décrit plus loin.

c) Détermination de l'acidité totale. - Au contenu du vase ajouter 10 gouttes de solution de phénolphtaléine à $1 \%$ et neutraliser avec une liqueur de soude $\mathrm{N}$ - noter le volume employé. Ajouter ensuite $0 \mathrm{~cm}^{3} 5$ de soude $\mathrm{N}$ pour avoir un léger excès de $\mathrm{NaOH}$ - noter le volume total employé.

Le premier chiffre permettra le calcul de l'acidité lactique. Le résultat diminué :

1. De l'acidité due au bichromate qui sera dosé ultérieurement;

2. De l'acidité normale du lait, puis rapporté à 1 kilogramme de lait, fera connaitre la correction à appliquer au chiffre du lactose dosé par la suite.

d) Mise en solution de l'échantillon. - Matériel nécessaire : entonnoir à séparation (allonge) bouché à l'émeri de 1 litre au moins, muni d'un robinet percé d'une lumière assez large. On y trace un repère de graduation à $230 \mathrm{~cm}^{3}$ environ. 
Réactifs nécessaires :

1. Liqueur d'Adam, de formule :

Cm. cubes

Ether sulfurique

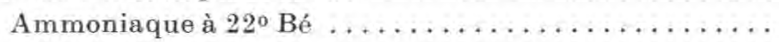

Alcool à $95^{\circ}$

Eau distillée $\ldots \ldots \ldots \ldots \ldots \ldots \ldots \ldots \ldots \ldots \ldots \ldots \ldots \ldots \ldots \ldots \ldots \ldots$

2. Ether de pétrole (densité : 0,65).

Faire passer la totalité de l'échantillon dans l'allonge. Calculer approximativement son volume, qui comprend :

Volume du lait déduit du poids + eau ajoutée $+\mathrm{NaOH}$ " $\mathrm{N}$ " $=\mathrm{V}$.

Prendre un volume de liqueur d'Adam $=\mathrm{V} \times 2,2$.

Utiliser cette liqueur d'Adam pour rincer à fond tout le matériel. On arrive facilement à rassembler sans perte tout l'échantillon dans l'allonge. Boucher celle-ci, la retourner, ouvrir le robinet (à cause de la surpression qui se produit au début). Agiter aveo précaution d'abord, puis énergiquement par la suite en s'y prenant à deux ou trois reprises pendant une minute chaque fois. Laisser reposer à $25^{\circ}$ environ.

$\mathrm{Au}$ bout de peu de temps (une heure au plus) la séparation est complète, on obtient à la partie inférieure une solution colloïdale bien homogène. Il nous est arrivé dans de vieux laits caillés de voir déposer au bas de l'allonge quelques grumeaux de caséine : ils ont été déchiquetés avec un long fil de fer, puis soutirés dans un mortier, bien broyés, délayés avee une petite quantité d'eau (et non pas de liqueur d'Adam, et réintroduits dans l'allonge.

Ensuite, on soutire complètement la partie aqueuse dans un ballon jaugé de $1.000 \mathrm{~cm}^{3}$ (ce volume est nécessaire si l'on opère sur $250 \mathrm{~cm}^{3}$ de lait).

On purifie la solution éthérée restée dans l'allonge en lui ajoutant un volume d'éther de pétrole $=\mathrm{V} / 2$ (voir plus haut) et quelques gouttes d'héliantine. Agiter énergiquement et laisser déposer. La partie inférieure colorée est jointe à la solution aqueuse. La solution éthérée contenant toute la matière grasse reste dans l'allonge.

e) Dosage de la matière grasse. - Le volume de solution éthérée est recueilli dans un ballon jaugé de $250 \mathrm{~cm}^{3}$ que l'on complète au trait. (Si ce volume restant dans l'allonge dépasse le repère de $230 \mathrm{~cm}^{3}$ qui y a été tracé, le plus simple paraît de réduire le volume d'éther en opérant ainsi : l'allonge est fermée par un bouchon de caoutchouc portant deux tubulures, l'une est ouverte à l'air, l'autre est reliée à une trompe à vide. L'aspiration produite entraîne la vapeur d'éther. On s'arrête lorsque la graduation : $230 \mathrm{~cm}^{3}$ est atteinte. Laisser l'éther se réchauffer un peu, transvaser dans un 
ballon jaugé de $250 \mathrm{~cm}^{3}$. Rincer à l'éther. Compléter au trait. Mélanger).

Le dosage de la matière grasse sera fait sur 20 au $25 \mathrm{~cm}^{3}$.

On en déduira la matière grasse par kilogramme de lait.

\section{Analyse de la solution aqueuse contenant les autres constituants}

Cette solution se trouve dans le ballon jaugé de $1.000 \mathrm{~cm}^{3}$. Compléter au trait avec de l'eau distillée. Le mélange demande quelques précautions : l'agitation produirait au début une surpression susceptible de causer des pertes. Il vaut mieux verser le liquide dans un vase assez grand et rendre homogène par agitation. On a ainsi $1.000 \mathrm{~cm}^{3}$ d'une solution $\mathrm{A}$, correspondant à un poids $\mathrm{P}$ de lait.

f) Dosage de l'extrait sec dégraissé. - Dans une capsule de platine, mesurer $20 \mathrm{~cm}^{3}$ de la solution A. Porter au bain-marie pendant sept heures (se méfier de la mousse qui se forme au début et chauffer d'abord lentement sur la plaque du bain-marie). Peser. Rapporter les résultats à un kilogramme de lait.

Le poids trouvé doit être corrigé :

$10 \mathrm{Du}$ bichromate ;

$2^{\circ}$ De la soude qui a été ajoutée au début.

L'acide lactique est neutralisé selon la réaction :

$\mathrm{CH}^{3}-\mathrm{CHOH}-\mathrm{CO}^{2} \mathrm{H}$

$\mathrm{CH}^{3}-\mathrm{CHOH}-\mathrm{CO}^{2} \mathrm{Na}$

90

112

On voit que la neutralisation de l'acide lactique par $\mathrm{NaOH}$ augmente le poids de sa molécule de 22. Donc chaque centimètre cube de $\mathrm{NaOH}$ ajoutée a contribué à augmenter l'extrait de 22 milligraimmes.

g) Dosage des matières minérales. - Caloiner l'extrait au rouge très sombre avec les précautions d'usage.

Correction à effectuer : $1^{\circ}$ Le bichromate ;

$2^{\circ} \mathrm{NaOH} \mathrm{N}$ ajoutée.

Le lactate de soude produit dans la neutralisation de l'acidité se transforme à la calcination en $\mathrm{CO}^{3} \mathrm{Na}^{2}$. Donc toute la soude ajoutée doit être déduite du poids des cendres en comptant que $1 \mathrm{~cm}^{3}$ de $\mathrm{NaOH} N=0$ gr. 053 de $\mathrm{CO}^{3} \mathrm{Na}^{2}$.

Ces corrections effectuées, on obtient le chiffre de matières minérales par kilogramme de lait.

h) Dosage du bichromate de $K$ sur les cendres. - Dans les laits fraîchement additionnés de bichromate de $K$, on peut doser le 
bichromate, soit sur les cendres, soit sur le liquide déféqué pour le dosage du lactose (voir à j).

Les résultats trouvés sont identiques.

Il n'en est pas de même si le lait est devenu "bleu " si le bichromate a été réduit en sel de chrome, en totalité ou en partie.

Pour connaître la quantité de bichromate non altéré, il faut faire le dosage sur le liquide déféqué.

Pour connaître le chrome total, effectuer le dosage sur les cendres; l'expérience montre que, même dans les laits très bleus où le bichromate est fortement réduit, la calcination ramène tout le chrome à l'état de chromate alcalin. Il est dosé dans les cendres par la méthode classique.

i) Matières azotées totales. - Il paraît, a priori, difficile de doser les matières azotées dans un milieu enrichi en $\mathrm{NH}^{3}$ par l'addition de liqueur d'Adam. Nous avons tourné cette difficulté d'une manière qui semble satisfaisante. Les essais répétés effectués sur un lait normal et sur le même lait vieilli, ayant subi le traitement décrit ci-dessus, ont donné des chiffres concordants, en opérant ainsi :

$20 \mathrm{~cm}^{3}$ de solution A sont mesurés dans une capsule de platine et portés au bain-marie pendant une heure, jusqu'à dessiccation. A ce moment, l'ammoniaque est complètement chassée; comme le lait avait été préalablement neutralisé par $\mathrm{NaOH}$ et, par précaution, additionné d'un léger excès de cet alcali, il ne reste pas d'ammoniaque en combinaison.

On pourrait, au contraire, objecter que cette manière de faire entraîne une perte d'ammoniaque et de bases azotées volatiles dans les laits très altérés, perte qui d'ailleurs ne doit pas être très importante en poids. Dans de tels laits, le chiffre trouvé devra être considéré comme minimum.

Le résidu de la eapsule de platine est repris par quelques centimètres cubes d'eau chaude, introduite dans un ballon en pyrex de $300 \mathrm{~cm}^{3}$ et le dosage de $\mathrm{N}$ est fait par la méthode Kjeldähl.

On obtient les matières azotées totales par kilogramme de lait.

Défécation de la solution A:

Mesurer exactement $100 \mathrm{~cm}^{3}$ de solution A. Les faire passer dans un ballon en pyrex de $500 \mathrm{~cm}^{3}$. Raccorder ce ballon à la trompe à vide et aspirer à froid pendant quelques minutes jusqu'à cessation de tout dégagement gazeux. On chasse ainsi tout l'éther dissous qui serait gênant par la suite.

Faire passer le liquide dans un ballon jaugé de $200 \mathrm{~cm}^{3}$. Neutraliser par l'acide acétique, versé goutte à goutte, puis ajouter 
$4 \mathrm{~cm}^{3}$ de ferro-cyanure de $\mathrm{K}$ à $15 \%$ et $4 \mathrm{~cm}^{3}$ d'acétate de zinc à $30 \%$. Compléter à 200. Mélanger, laisser en contact 5 minutes. Filtrer. On obtient une liqueur B sur låquelle on pourra doser, bichromate de $\mathrm{K}$ non altéré, lactose et chlorures.

j) Dosage de bichromate de $K$. - Prendre $50 \mathrm{~cm}^{3}$ de liqueur B, y ajouter $3 \mathrm{~cm}^{3}$ de $\mathrm{Kl}$ à $10 \%$, puis $4 \mathrm{~cm}^{3} \mathrm{de} \mathrm{HCl}$ pur. Titrer l'iode mis en liberté par Hypo N/10. Ce dosage fournira la quantité de bichromate conservé dans le lait sous cet état et pourra donner une idée de son degré d'altération.

k) Dosage de lactose. - Il faut commencer par éliminer le bichromate. Pour cela, au reste de la liqueur B, ajouter de l'acétate de plomb en poudre en quantité très petite (quelques milligrammes) juste suffisante pour transformer le bichromate en sel de plomb, ce qui se constate par la disparition de la coloration jaune du liquide décanté. Après quelques minutes de contac $`$, filtrer. On a une liqueur B' qui doit être incolore.

Le lactose est dosé par la méthode de Bertrand sur $10 \mathrm{~cm}^{3}$ de liqueur B' $+10 \mathrm{em}^{3}$ d'eau $+20 \mathrm{~cm}^{3}$ de chacune des-liqueurs A et B de Bertrand. On exprime les résultats en lactose hydraté par kilogramme de lait et on ajoute au chiffre trouvé la correction d'acidité déterminée comme il est indiqué en e (voir plus haut).

\section{Remarques :}

I. Si l'on ne veut pas doser le bichromate sur la liqueur B, il est plus simple d'ajouter au liquide déféqué par le ferrocyanure de zinc avant filtration quelques gouttes - 5 à 10 - d'acétate basique de plomb Codex en quantité juste suffisante pour éliminer le bichromate. Cette manière de faire nous a toujours donné de bons résultats.

II. Si l'on pense à une hydrolyse possible du lactose, il sera bon de faire un dosage après hydrolyse complète, au moyen de HCl chloruré, comme il est indiqué dans Denrgès, Chelle et Labat (Précis de Chimie analytique, 6e édition, t. II, page 384).

Il est préférable de déterminer soi-même le coefficient à employer pour revenir du lactose hydrolysé au lactose hydraté initial en partant d'une solution titrée de lactose traitée dans les mêmes conditions que le lait analysé. En utilisant la méthode de Bertrand, nous avons trouvé :

Pouvoir réducteur du lactose hydraté $\times 1,43=$ Pouvoir réducteur du lactose hydrolysé.

III. En fait, quand l'hydrolyse du lactose est un peu avancée, il est bien difficile d'avoir des résultats exacts. 
Voici un exemple :

LAIT MOUILlé ET BICHROMATÉ, PRÉleVÉ LE 9 OCTOBRE

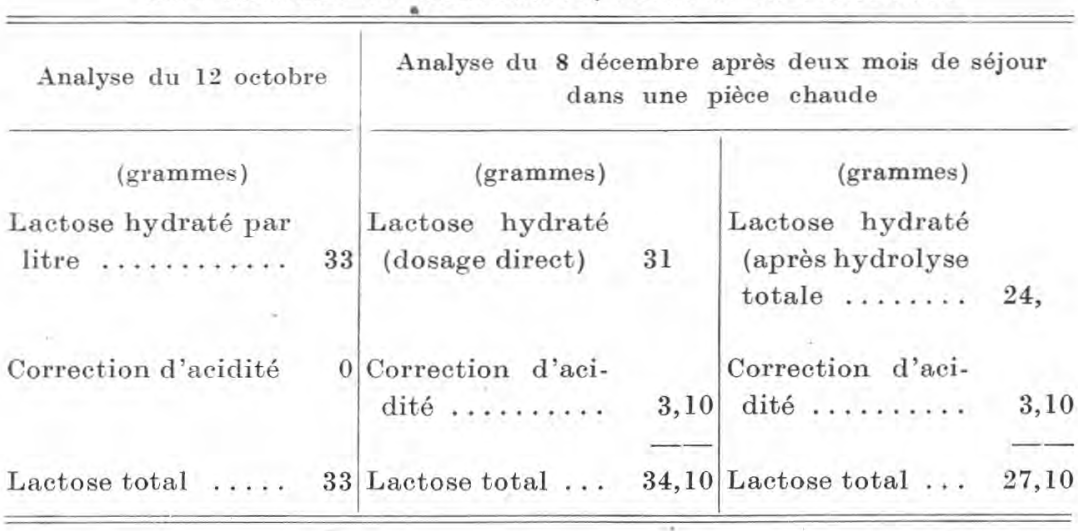

Le dosage direct du 8 décembre donne un chiffre un peu trop élevé, mais le dosage du lactose après hydrolyse donne un chiffre trop faible.

On peut admettre que dans les laits "bleus » âgés :

Une partie du lactose est hydrolysée ;

Une partie du lactose a été transformée en acides que l'on évalue en acide lactique ;

Une autre partie a été détruite par fermentation dont les résidus ne sont pas titrés, si bien que le chiffre trouvé n'est qu'un compromis plus ou moins approché de la réalité, quelle que soit la méthode employée.

En s'éclairant de l'exemple ci-dessus, il paraît logique et prudent dans une expertise de lait altéré de s'en tenir aux résultats obtenus par le dosage direct, sans tenir compte de l'hydrolyse (même si le chiffre trouvé risque d'être un peu trop élevé).

1. Dosage des chlorures. - Il se fait par la méthode de Charpentier-Volhard, en opérant sur $50 \mathrm{~cm}^{3}$ de la liqueur B' $+5 \mathrm{~cm}^{3}$ $\mathrm{NO}^{3} \mathrm{AgN} / 10+5 \mathrm{~cm}^{3} \mathrm{NO}^{3} \mathrm{H}$ pur exempt de vapeurs nitreuses $+2 \mathrm{~cm}^{3}$ d'alun de fer ammoniacal à $25 \%$. - On titre par KSCN $\mathrm{N} / 10$.

On calcule en $\mathrm{NaCl}$ par kilogramme de lait.

m) Extrait total. - Il suffit de faire la somme de tous les éléments dosés. Son exactitude dépendra surtout de celle du chiffre de lactose. Si le dosage du lactose est faussé par suite d'altération, il est évident que l'extrait le sera aussi.

On rapporte les résultats à 1 kilogramme de lait.

n) Calcul de la densité du lait initial. - Il est difficile de prendre 
le volume exact de l'échantillon, mais on peut déterminer la densité d'une manière assez précise avec la formule suivante :

$$
(\mathrm{D}-1)
$$

$$
\mathrm{E}=2.665 \ldots+1,2 \mathrm{~B} \quad \text { (formule } \mathrm{n}^{\circ} 2 \mathrm{de} \text { Fleischmann) }
$$

Dans laquelle D est la densité du lait ;

$B$ est la matière grasse par kilogramme ;

$\mathrm{E}$ est l'extrait total par kilogramme.

$\mathrm{B}$ et $\mathrm{E}$ sont connus; ils ont été déterminés.

Il serait encore plus simple d'utiliser le disque calculateur d'Ackermann qui est gradué en se basant sur cette formule de Fleischmann (Pien, Le Lait, 1947, p. 332).

On ne peut prétendre trouver une densité exacte que pour leslaits peu altérés.

o) Résultats exprimés par litre de lait. - Connaissant la quantité de chaque élément par kilogramme et la densité, on ramène les résultats au litre de lait.

p) Constante moléculaire simplifiée. - Sa détermination ne présente aucune difficulté, sous la réserve, maintes fois répétée, que le chiffre de lactose puisse inspirer confiance.

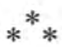

\section{Degré de précision de la méthode}

Voici, prises parmi plusieurs, les analyses de deux laits faites d'abord par la méthode ordinaire sur le lait en bon état, et ensuite, après altération, sur la totalité de l'échantillon restant (150 grammes environ).

\begin{tabular}{|c|c|c|}
\hline \multicolumn{2}{|c|}{$\begin{array}{c}\text { Analyse du jour de la collecte } \\
\text { du } 16 \text { novembre } \\
\text { (méthode ordinaire) }\end{array}$} & $\begin{array}{l}\text { Analyse du lait } \\
\text { complètement caillé } \\
\text { le } 8 \text { décembre } \\
\text { (sur tout l'échantillon) }\end{array}$ \\
\hline$\ldots \ldots \ldots \ldots \ldots$ & 1. 031,2 & 1.030 \\
\hline Matière grasse ............. & 43,40 par litre & 43,05 par litre \\
\hline Matières azotées .......... & $37,50-$ & $37,40-$ \\
\hline Lactose hydraté $\ldots . \ldots \ldots \ldots$ & 50,60 & 31,70 \\
\hline Correction d'acidité $\quad \ldots \ldots \ldots$ & - & 9,10 \\
\hline Cendres $\ldots \ldots \ldots \ldots \ldots \ldots \ldots$ & 7,70 & 7,90 \\
\hline Chlorure de sodium $\ldots \ldots \ldots$ & 1,70 & 1,73 \\
\hline
\end{tabular}

I. LAIT FRAIS - SANS ADDITION D'ANTISEPTIQUE

Concordance satisfaisante pour tous les dosages, sauf le lactose. 
2. LAIT BICHROMATÉ ET MOUILLÉ, PRÉLEVÉ LE 8 OCTOBRE 1950

\begin{tabular}{|c|c|c|}
\hline \multicolumn{2}{|c|}{$\begin{array}{l}\text { Analyse du } 12 \text { octobre } \\
\text { (méthode ordinaire) }\end{array}$} & $\begin{array}{l}\text { Analyse du } 8 \text { décembre } \\
\text { après séjour dans } \\
\text { une pièce chaude } \\
\text { (sur tout l'échantillon) }\end{array}$ \\
\hline Densité $\ldots \ldots \ldots \ldots \ldots \ldots$ & $1.023,0$ & $1,022,3$ \\
\hline Matière grasse . . . . . . . . . & 34 par litre & 33,70 par litre \\
\hline Matières azotées .......... & $24,80-$ & $24,40-$ \\
\hline Lactose hydraté . . . . . . . . . & 33 & $34,10(1)-$ \\
\hline Cendres $\ldots \ldots \ldots \ldots \ldots \ldots \ldots$ & 5,40 & $5,60-$ \\
\hline Chlorure de sodium $\ldots . \ldots \ldots$ & 1,20 & $1,26-$ \\
\hline
\end{tabular}

Les chiffres sont très voisins, même ceux de lactose. Et pourtant - le 8 décembre, il y avait une forte hydrolyse compensée par la destruction partielle du glucose et du galactose formés.

Il est bien évident que ce chiffre de lactose ne pourra être interprété qu'avec une extrême prudence en ne perdant pas de vue le degré d'incertitude qu'il comporte.

Remarque. - On peut reprocher à cette méthode de consommer une grande quantité de réactifs. En fait, pour un échantillon de lait de $250 \mathrm{~cm}^{3}$, elle exige environ $300 \mathrm{~cm}^{3}$ d'éther, $200 \mathrm{~cm}^{3}$ d'alcool et $125 \mathrm{~cm}^{3}$ d'éther de pétrole. Il est possible toutefois de récupérer une bonne partie de ces solvants, il suffit d'agiter la solution éthérée contenant la matière grasse avec quelques centimètres cubes d'eau acidulée par $\mathrm{SO}^{4} \mathrm{H}^{2}$ pour fixer le peu d'ammoniaque restant dans l'éther, et d'effectuer une distillation fractionnée.

\section{Conclusions}

Il est bien certain que la technique qui vient d'être exposée ne se prête pas au travail en grande série et ce n'est pas son but. Son emploi se justifie surtout pour un dosage précis de matière grasse dans un lait baratté ou coagulé quand une analyse est absolument nécessaire : lait suspect, aveu de falsification, nécessité d'une expertise. Elle permet, comme nous l'avons vu par les exemples cités, une analyse complète suffisamment précise quand le lait est encore en bon état, et un dosage assez exact de tous les éléments, sauf le lactose, dans les échantillons mal conservés. Elle est peut-être susceptible de rendre quelque service aux experts qui reçoivent souvent des échantillons dans un état de fraîcheur laissant beaucoup à désirer. Présente-t-elle un progrès sur les méthodes employées jusqu'ici pour l'analyse des laits altérés ? Il est peut-être téméraire

(1) Dosage direct, correction faite de l'acidité. 
de le penser. Quoi qu'il en soit, c'est pour essayer de rendre serviceà nos collègues chimistes que nous nous sommes décidé à la leur. communiqer.

\title{
L'INDUSTRIE LAITIÈRE DANS LE MONDE
}

\author{
par \\ G. GÉNIN \\ Ingéniour E.P.C.
}

\section{ANGLETERRE}

\section{Nouveau produit pour la fabrication de la crème glacée}

Ce nouveau produit, dénommé Néolac, peut être utilisé par les fabricants de crème glacée, en remplacement de la poudre de lait et du lactose. Il s'obtient par séchage par atomisation de petit-lait et se présente sous la forme d'une poudre blanche, soluble dans l'eau, qui renferme $30 \%$ de lactose et $1 \%$ d'amidon.

Il peut être également utilisé par les fabricants de saucisses,. de pâtisseries et de confiseries, et par exemple, additionné dans la proportion de $6 \%$ dans les mélanges employés pour la fabrication des saucisses, il remplace avantageusement la poudre de lait, demême que sa haute solubilité le rend intéressant dans d'autres industries alimentaires.

Si l'on veut utiliser un produit de haute valeur nutritivè, le Néolac est livré en qualité S qui renferme $24 \%$ de lactose, $24 \%$ de protéine, $29 \%$ de maltose, et $9 \%$ de graisse.

\section{Nouvelle méthode normalisée pour l'analyse chimique du beurre}

La British Standards Institution vient de publier récemment une nouvelle édition de sa norme BS 769, intitulée "Méthodes pour l'analyse chimique du beurre ». La première édition datait de 1938, la nouvelle a été modifiée et comporte en particulier comme additions des méthodes de dosage du sel, de l'acidité titrable, du fer et du cuivre.

Par ailleurs, les procédés qui figuraient déjà dans l'édition ancienne ont été revus et portent sur le dosage de la graisse, des acides volatils, la détermination de l'indice d'iode, de l'indice de saponification et de l'indice de réfraction. Rappelons enfin que le document décrit également des méthodes de préparation d'échantillons, en vue de la détermination de l'humidité, du sel, de la teneur en graisse et de la concentration des ions $\mathrm{H}$. On peut se pro- 